\title{
Long-term prognosis for atypical and malignant meningiomas: a study of 71 surgical cases
}

\author{
Lucio Palma, M.D., Paolo Celli, M.D., Carmine Franco, M.D., Luigi Cervoni, M.D., and \\ Giampaolo Cantore, M.D.
}

Department of Ophthalmological and Neurosurgical Sciences of Siena University Medical School, Siena, Italy; and Department of Neurological Sciences of Rome University "La Sapienza" Medical School, Section of Neurosurgery, Rome, Italy

To contribute to a better understanding of the prognostic differences between atypical and malignant meningiomas as defined by the World Health Organization (WHO) and the influence of the grade of initial surgical excision on postoperative course, 42 cases of atypical and 29 of malignant meningioma were studied, along with long-term follow up. The two groups were compared with respect to long-term survival, recurrence-free survival, and median time to recurrence. The prognostic significance of the Simpson grade of surgical resection and tumor location was also considered. Survival at 5 and 10 years was recorded in $95 \%$ and $79 \%$, respectively, of patients with atypical meningioma and in $64.3 \%$ and $34.5 \%$ of patients with malignant meningioma $(\mathrm{p}=0.001)$. Recurrence-free survival and median time to recurrence were also significantly longer in patients with atypical than in those with malignant meningiomas: 11.9 versus 2 years $(p=0.001)$ and 5 versus 2 years $(p<0.0041)$, respectively. Six $(26 \%)$ of the 23 recurring atypical meningiomas became malignant. Simpson Grade I resection and location in the cerebral convexity, which were closely related, were found to be associated with a significantly better clinical course in the entire series ( $\mathrm{p}^{3 / 4}$ 0.0016). Patients with atypical meningiomas fared better than those with malignant meningiomas after incomplete surgical excision (Simpson Grades II-III), but the difference was not statistically significant. Multivariate analysis using the Cox model indicated that radical extirpation (Simpson Grade I vs. II-III) and histological findings (atypical meningioma vs. malignant meningioma) were significantly related to prolonged survival ( $\mathrm{p}<0.0003$ and $\mathrm{p}<0.0388$, respectively). In conclusion, the current study shows that for most patients with atypical meningioma the prognosis was less severe than for those with malignant meningioma, but the risk of a downhill course resulting from malignancy after incomplete resection and recurrence was not negligible (26\%). In addition, the WHO classification was found to be inadequate for a minority of the atypical meningioma cases, which currently have the same unfavorable course as cases of malignant meningioma. The results also indicate that objective Simpson Grade I extirpation of convexity meningiomas can be successful despite histological findings of malignancy.

Key Words * atypical meningioma * malignant meningioma * long-term prognosis

The existence of atypical and malignant meningiomas was clearly recognized by Cushing and Eisenhardt[8] in 1938. In their classic monograph they described a variant of meningioma characterized 
by a papillary pattern of epithelioid cells or sheeting of polyhedral, round, and fusiform cells with abundant mitoses and possible brain infiltration. They grouped these cases as Type $\mathrm{V}$, variant 1 , and stressed the poor long-term prognosis of these meningiomas by describing the histories of two patients. At the same time, however, they noted that five of 10 patients in this group with "atypical" meningiomas had postoperative survival times of more than 5 years. The prognosis for this group was therefore less favorable than for the group with benign meningiomas, but was better than that for patients in a subsequent group classified with Type VI malignant meningioma, who had a mean survival time of 2.5 years.[8] It was subsequently suggested by other authors that the malignant meningiomas could be further divided into low- and high-malignancy types.[35,36,41] This division was adopted by the World Health Organization (WHO) in its first and recently revised second edition classifications of meningiomas, with two grades of malignancy termed atypical (Grade II) and anaplastic (Grade III).[21,40] Unfortunately, the WHO classification gives mostly qualitative criteria in defining atypical and anaplastic meningiomas and does not suggest more precise quantitative indications such as numerical scoring systems.[21,24] As a consequence, detailed correlative clinicopathological studies on this subject are difficult to perform and it is not possible to compare data from different centers. In particular, the clinical behavior of atypical meningiomas, regarded as less severe than malignant tumors and intermediate between benign and malignant forms, must still be precisely defined by further clinicopathological studies.[2,6,12,14-16,23,24,39,41] Moreover, the prognostic influence of radical surgery on outcome has not been discussed as thoroughly for atypical and malignant meningiomas[2,5,16,24,30,34,39] as for benign meningiomas.[1,14,25,27,31,33] The radicality of surgical excision, in turn, depends mainly on the meningioma site and was graded objectively by Simpson[33] in his well-known paper, in which he pointed out that total removal was sometimes only subjectively assessed by the surgeon and that this gave rise to reports of a $9 \%$ recurrence rate after so-called "complete excision" of benign meningiomas. This percentage can reasonably be expected to be higher for anaplastic meningiomas. To provide additional information on these questions, we reviewed the cases of atypical and malignant meningioma in our series, considering also the tumor site and Simpson grade of the initial surgical resection. We evaluated the influence of histological and anatomicosurgical factors on long-term survival, recurrence-free survival, and the time interval to recurrence.

\section{CLINICAL MATERIAL AND METHODS}

We reviewed our series of 1253 intracranial meningiomas treated surgically between 1951 and 1986 and selected 108 cases (8.6\%) classified as atypical or anaplastic (that is, malignant); these form the basis of the present study. Meningeal hemangiopericytomas were excluded and will be discussed in a separate paper. The 108 cases included 61 atypical and 47 anaplastic meningiomas. To provide more precise and more comparable data than in our previous study,[28] we reviewed all histological sections randomly, without reference to the previous diagnosis or to outcome, and reclassified atypical and malignant meningiomas according to WHO guidelines. We also used a scoring system similar to that created by Jääskeläinen, et al.,[15,16] based on the following histological features: 1) hypercellularity/loss of architecture (described as "sheeting" by some authors); 2) nuclear pleomorphism; 3) high mitotic index; 4) necrosis; and 5) brain infiltration (Fig. 1). As suggested by Kepes,[19] we also added the papillary pattern that we have seen as an occasional focus or extensively present throughout the tumor regardless of the type of meningioma. The severity of each histological parameter was estimated subjectively and graded from 0 to 3. Brain infiltration by itself was found to be a marker of malignancy; it was never found in atypical meningioma but was always associated with other severe histological features so that 
the score remained within the limits of malignancy. However, for the purpose of our scoring system it was evaluated as either present (3) or absent (0). Tumors scoring a total of 3 to 8 were classified as atypical meningiomas and those with a score of 9 to 18 as malignant meningiomas. As a result, 61 meningiomas were scored as atypical and 47 as malignant (anaplastic). The completeness of surgical resection was deduced from the description of the operation and, in more recent cases, by postoperative computerized tomography and nuclear magnetic resonance studies. However, for the purposes of the present study, Simpson Grade I cases were kept separate and Simpson Grade II cases were grouped with Grade III cases because of the small number of the latter. Also, unlike others, $[6,14,20,23]$ we regard Simpson Grade II surgery as an incomplete excision that cannot be expected to impede recurrence, especially of rapidly growing neoplasms.[14] In support of this view, the clinical courses of our patients with Simpson Grades II and III were practically the same. The distinction between Simpson Grade I and II resection was based on the objective Simpson criteria as reported by the surgeon in each case. Tumor sites were divided into three categories: convexity, parasagittal, and basal. In this series there was only one meningioma of the posterior fossa; it was a malignant cerebellopontine angle tumor invading the petrous bone.

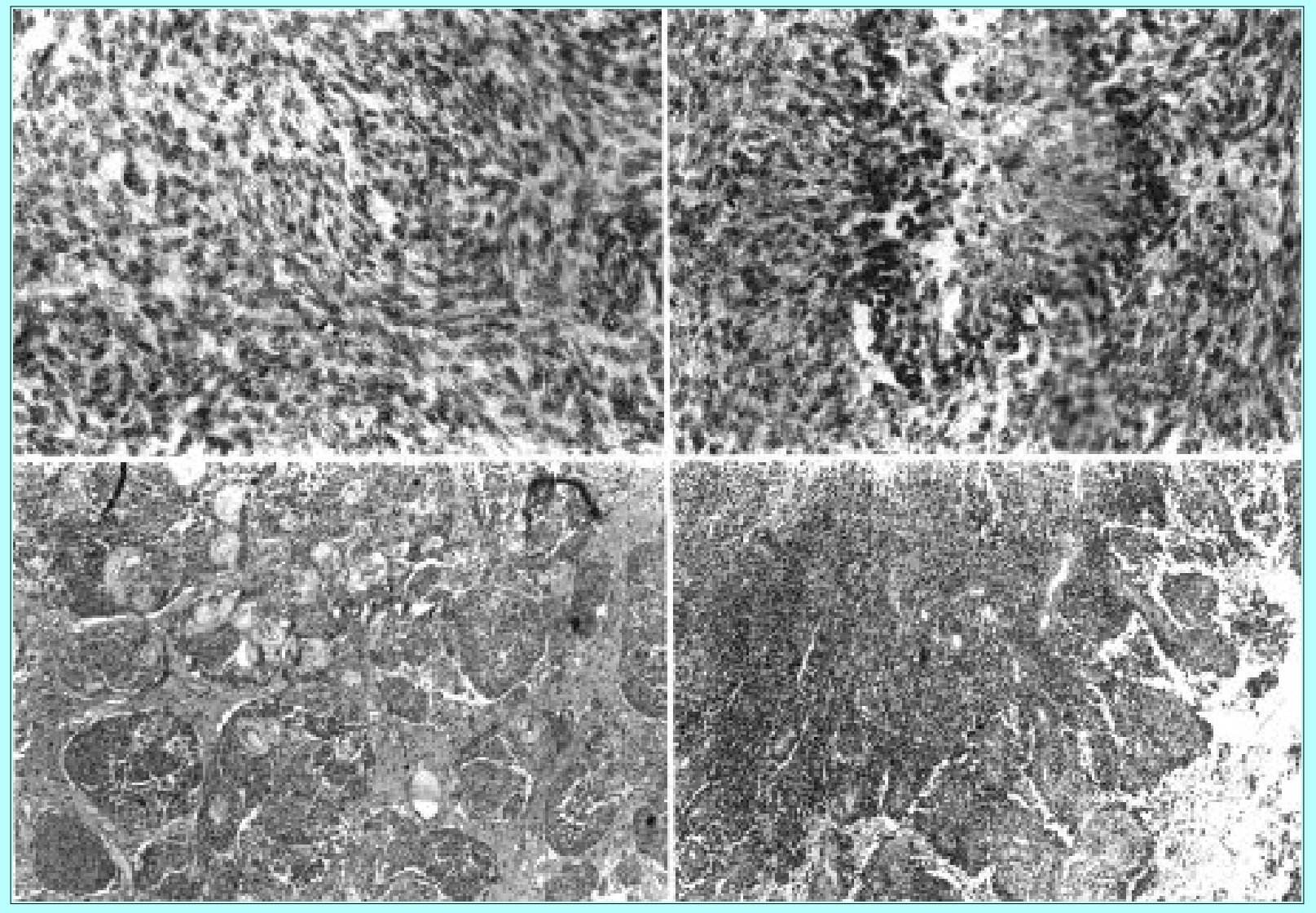

Fig. 1. Photomicrographs showing the main histological features of atypical and malignant meningiomas. Upper Left: Hypercellularity, sheeting, and mitoses. Upper Right: Necrosis and mitoses. Lower Left: Brain invasion. Lower Right: Papillary pattern. H \& E, original magnification X 200.

The clinical records of each patient were reviewed to obtain data on long-term survival, recurrence-free survival, and median time to recurrence, as well as Simpson grade of resection. The criterion for recurrence was detection of recurrent tumor by neuroradiological investigations prompted by recrudescence of neurological symptoms. When necessary, a special follow-up examination or telephone interview was requested. The follow-up period ended in 1994. 


\section{Statistical Analyses}

Statistical analysis for survival and recurrence-free survival was performed by comparing computer-generated curves estimated by the Kaplan-Meier method.[18] Survival and recurrence-free survival curve differences were assessed by log-rank tests (Breslow, Mantel-Cox, Tarone-Ware, and Peto-Prentice tests). Multivariate forward stepwise linear regression analysis was based on Cox's hazard function.[7] Comparisons of actuarial survival and recurrence-free rates at 5 and 10 years were based on chi-square tests or Fisher's exact test. Computations for curves and Cox models were performed using commercially available software.[10]

The prognostic influence of histological findings (atypical vs. malignant), Simpson grade of resection (Grade I vs. Grades II-III), and site (convexity vs. others) was investigated by multivariate analysis and by comparing appropriate cases in each histological group. Postoperative radiation therapy was used in a few cases, but because the protocols were different no reliable comparison can be made.

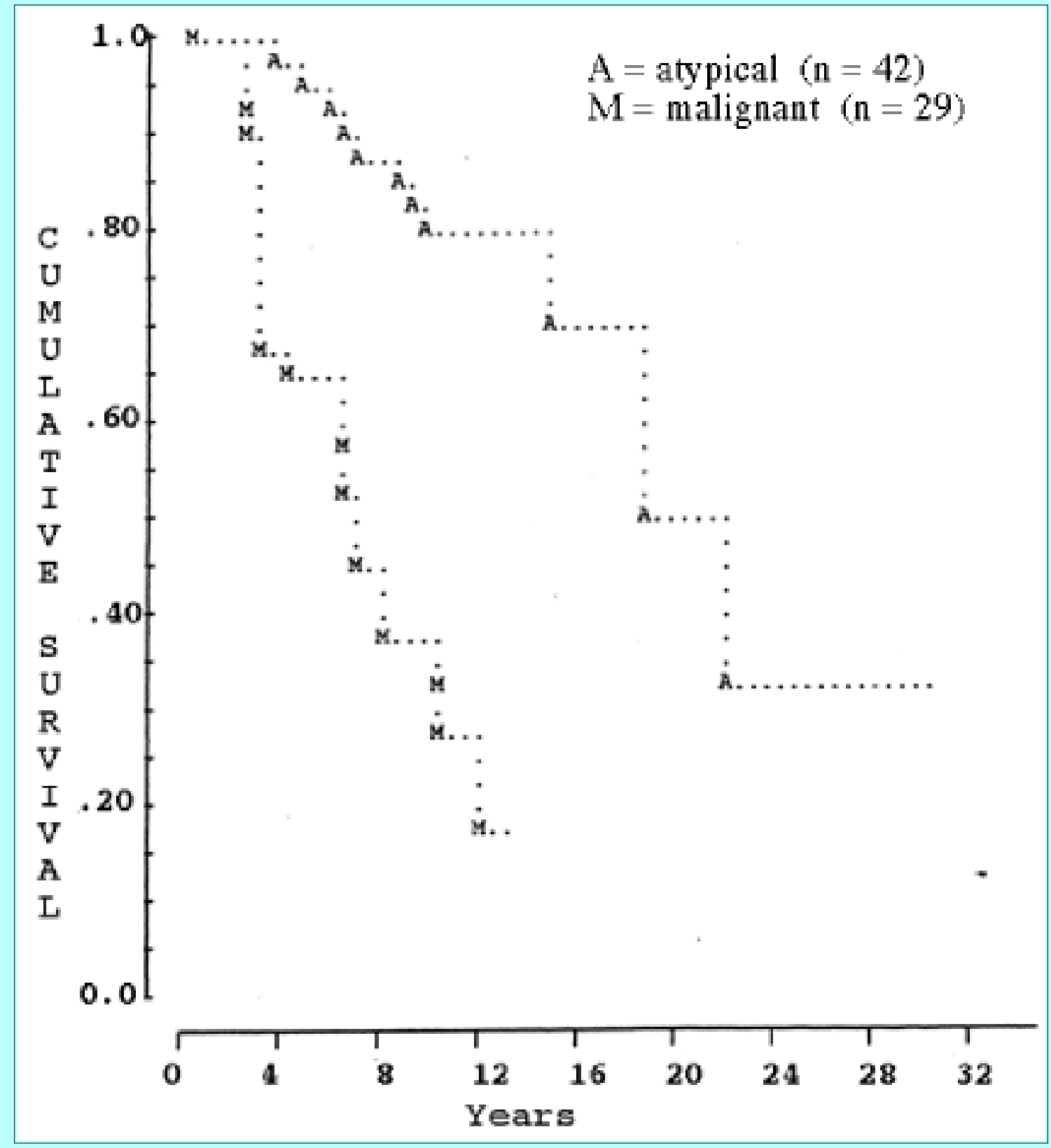

Fig. 2. Graph showing survival curves for patients who underwent surgery for nonbenign meningiomas. Atypical versus malignant: median survival time 19.01 versus 6.89 years 
according to the Breslow, Mantel-Cox, Tarone-Ware, and Peto-Prentice log-rank tests ( $\mathrm{p}=$ 0.0001).

\section{RESULTS}

\section{Atypical Meningiomas}

Nineteen of the 61 patients with atypical meningiomas were lost to follow-up review soon after the first operation. Of the remaining 42 patients ( 21 men and 21 women) 12 had convexity, 19 had parasagittal, and 11 had basal meningiomas. Surgical resection was described as Simpson Grade I in 14 cases (33.3\%) and Grades II to III in the other 28 cases. The median survival time was 19.01 years and 5- and 10-year survival rates were $95 \%$ and $79 \%$, respectively (Fig. 2). The median recurrence-free survival time was 11.93 years, with $77 \%$ and $55 \%$ of patients alive without recurrence at 5 and 10 years postsurgery, respectively (Fig. 3).

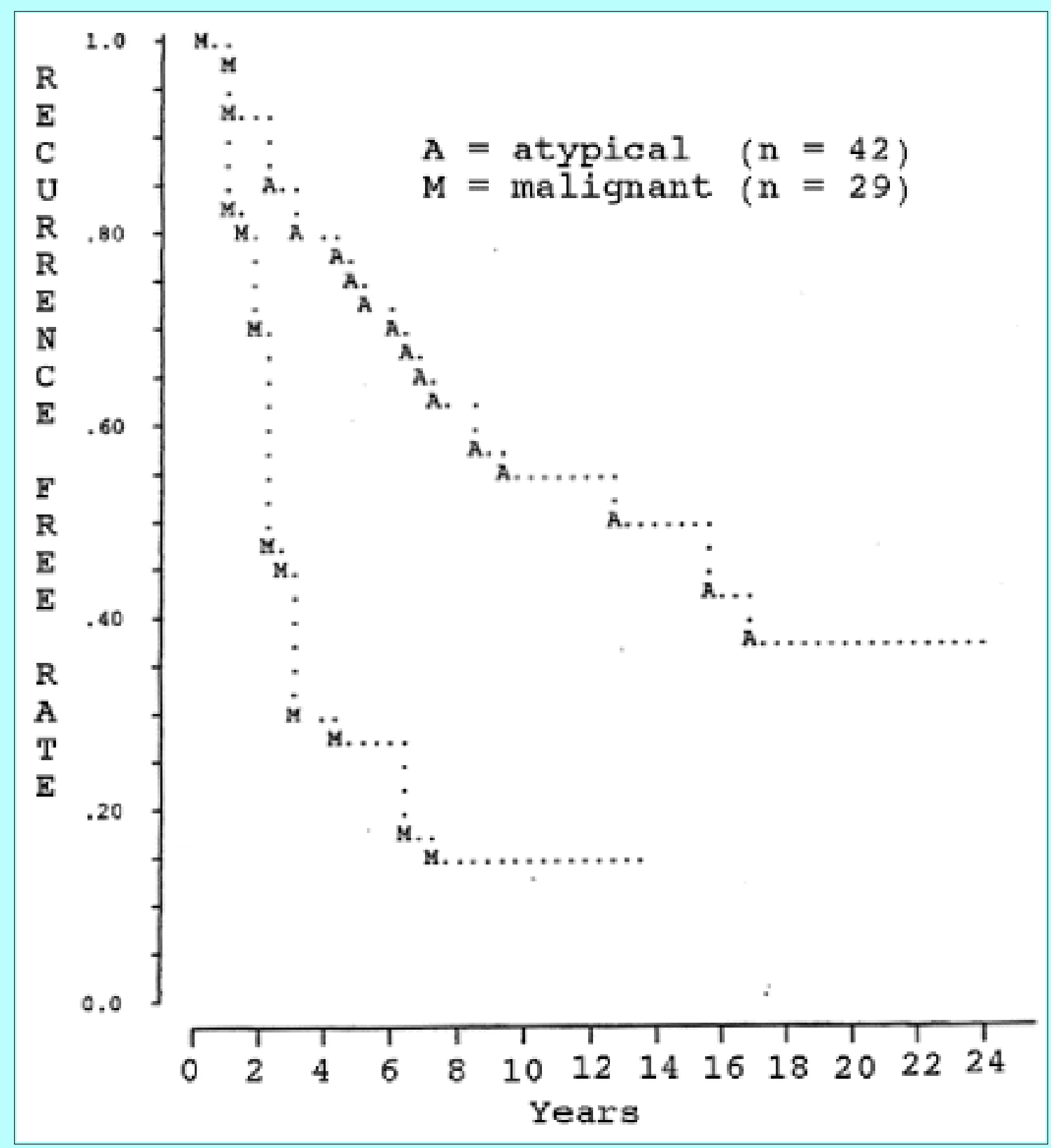

Fig. 3. Graph showing recurrence-free survival curves after the first surgery in 42 patients who underwent operation for atypical versus 29 for malignant meningiomas. The median 
recurrence-free survival time was 11.93 years for atypical versus 2 years for malignant meningiomas according to the Breslow, Mantel-Cox, Tarone-Ware, and Peto-Prentice log-rank tests $(\mathrm{p}=0.0001)$.

The recurrence rate was $35 \%$ and $52 \%$ at 3 and 5 years, respectively, and the median time to the first recurrence was 5 years (range 10 months-16 years) (Fig. 4). The number of recurrences per patient varied from one to six, with a mean of two. Late mortality due to recurrence was observed in 13 patients (31\%), 3.5 to 22.5 years (mean 10.5 years) after the initial surgery. Radiotherapy (4500-6500 cGy in 25-33 fractions) was administered in six cases: after the first operation in one patient who was alive without recurrence 15 years later and after the second operation in five patients, four of whom had died by 10 years after the first operation, and the other of whom had a recurrence at 10 years. Survival rates were significantly better after Simpson Grade I resection than after Grades II to III ( $p<0.0071)$. The best clinical course was observed in nine patients with Grade I extirpation of convexity meningiomas who were all alive without recurrence 10 to 19 years after the operation. Tumor location in the convexity was also associated with better survival than other sites but this was clearly secondary to the close correlation with Simpson Grade I extirpation $(r=0.75)$. It is noteworthy that in six $(26 \%)$ of the 23 recurrent cases there was a worsening of the histological picture from atypical to malignant: this was observed 6 to 15 years after the first operation with multiple recurrences in five patients, whereas tumor regrowth had become malignant by the second operation (after 8 years) in one patient. 


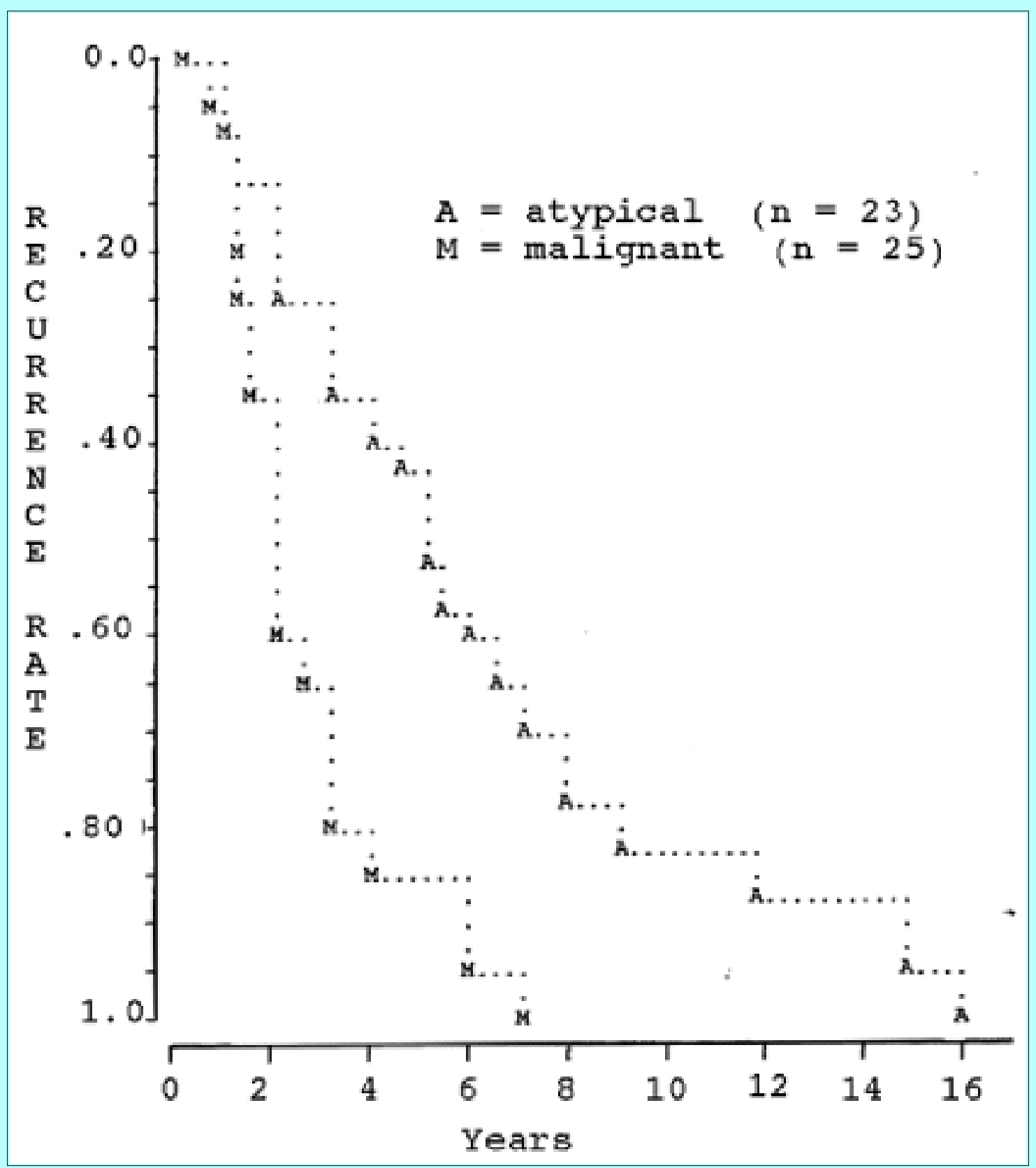

Fig. 4. Graph showing time to first recurrence in 23 atypical versus 25 malignant meningiomas. The median time to recurrence was 5 years for atypical versus 2 years for malignant meningiomas according to the Breslow, Mantel-Cox, Tarone-Ware, and Peto-Prentice log-rank tests $(\mathrm{p}<0.0041)$.

\section{Malignant Meningiomas}

Of the 47 patients with malignant meningioma (26 men and 21 women), 18 were lost to follow-up review immediately after the first operation. The site of the remaining 29 malignant meningiomas was as follows: 15 parasagittal, eight basal (one in the posterior fossa), and six convexity. Surgical resection was described as Simpson Grade I in six cases (21\%) and Grades II to III in the other 23 cases. The median survival time was 6.89 years and the actuarial 5- and 10-year survival rates were $64.3 \%$ and $34.5 \%$, respectively (Fig. 2). The median recurrence-free survival time was 2 years, with $45 \%$ and $15 \%$ of patients still alive without recurrence at 5 and 10 years, respectively (Fig. 3). The recurrence rates were $80 \%$ and $84 \%$ at 3 and 5 years, respectively, and the median time to recurrence was 2 years (range 7 months-7 years) (Fig. 4). Late mortality due to recurrence was recorded in 20 cases (69\%) 2 to 12 years 
after the first operation (mean 5.5 years). Radiation therapy (with the same modalities as for atypical meningioma cases in five patients and in the form of radiosurgery in two patients) was used in seven of these 20 patients (after the first recurrence in six) who had a mean survival time of 5.7 years (range $2-10.5$ years); the mean survival time of the 13 patients not receiving radiation therapy was 5.3 years (range 2-12 years). The survival time of six patients after Simpson Grade I extirpation was better than in the other 23 who underwent Grades II to III resection, but the difference was not statistically significant ( $p>0.079$ ). On the other hand, the six patients with convexity meningioma fared significantly better than the 23 with basal-parasagittal malignant tumors ( $\mathrm{p}$ < 0.0039). In the malignant meningioma group, as in the atypical meningioma group, there was a close correlation between location in the cerebral convexity and Simpson Grade I extirpation $(r=0.65)$. Multivariate analysis of the 29 malignant meningioma cases confirmed that location of the tumor in the cerebral convexity was the only positive factor for survival ( $\mathrm{p}$ $=0.0137$ ). The failure of radical extirpation (Simpson Grade I) to prolong survival significantly in patients with malignant meningiomas can be explained by the fact that of the six cases treated in this way, four were convexity and two were parasagittal meningiomas. Whereas all four patients with convexity meningiomas were alive without recurrence after a mean of 11 years, the two with parasagittal tumors died from recurrences after 2 and 6.5 years, respectively. Therefore we can reasonably assume that a "true" radical extirpation (that is, Simpson Grade I) was achieved in the four convexity but not in the two parasagittal meningioma cases.

\section{Combined Group}

As shown in Fig. 2-4, cumulative survival, recurrence-free survival, and median time to recurrence were all significantly better in patients with atypical than malignant meningioma. 


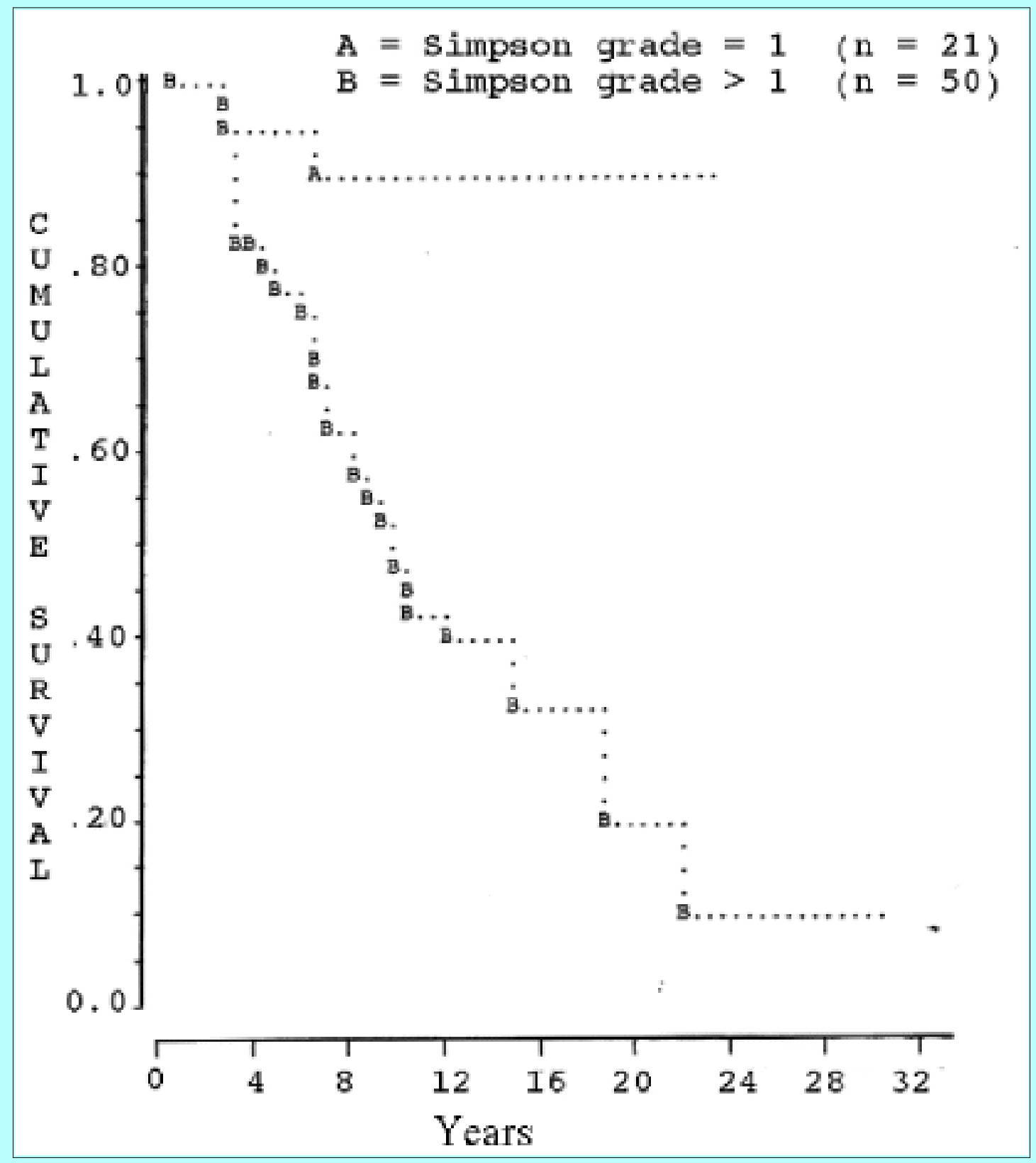

Fig. 5. Graph showing survival curves for 71 patients who underwent operation for nonbenign meningiomas. For Simpson grades of tumor removal of I versus II to III, the median survival time was not calculable versus 9.25 years according to the Breslow, Mantel-Cox, Tarone-Ware, and Peto-Prentice log-rank tests ( $\mathrm{p} 3 / 4$ 0.0016).

The favorable prognostic influence of radical surgical resection is demonstrated by the significantly better survival curve of patients undergoing Simpson Grade I excision of their tumor as compared to those who underwent Simpson Grades II to III excision (Fig. 5). The relationship of histological and surgical factors to the prognosis is further illustrated by the fact that, after incomplete surgical excision (Simpson Grades II-III) patients with atypical meningioma had a better survival curve than patients with malignant meningioma, but the difference was not statistically significant (Fig. 6). 


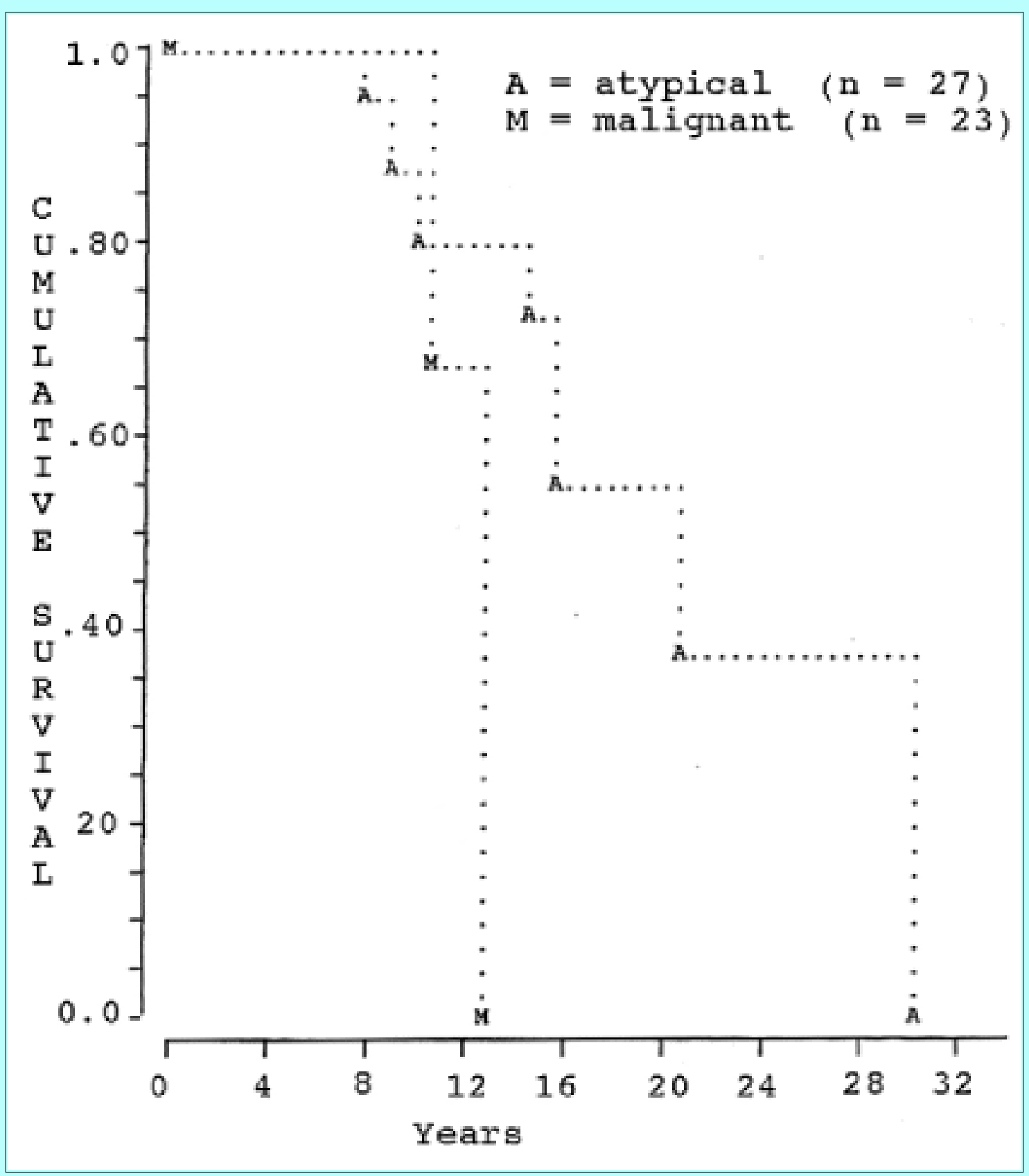

Fig. 6. Graph showing survival curves for patients who underwent operation for nonbenign meningiomas after Simpson Grades II to III tumor removal. Atypical versus malignant: median survival time 17.34 versus 11.50 years according to the Breslow, Mantel-Cox, Tarone-Ware, and Peto-Prentice log-rank tests ( $\mathrm{p}>0.3462$; not significant).

Multivariate analysis (Cox model) of factors positively influencing survival confirmed the significance of radical extirpation (Simpson Grade I) and histology (Table 1). 
TABLE 1

MULTIYRR ATE FORWARD STEPWISE LINEAR REGRESSION SURVNAL ANALYSIS,

PROBABLITY YALUES, AND PR IN 71 PATIENTS WHO UNDERWENT SURGERV

FOR ATVPICAL AND MAL GNANT MEN NGOMAS*

\begin{tabular}{llcc}
\hline \hline \multicolumn{1}{c}{ Factor } & \multicolumn{1}{c}{ Variables } & p Value & RR \\
\hline age & S45 ws $>45$ yrs & NS & 1.02 \\
Sex & Mis F & NS & 1.05 \\
tumor location & contexity ws others & NS & 3.89 \\
histological finding & atypical ws malignant & 0.0003 & 4.52 \\
surgical removal & Simpson Grade I ws II-III & 0.0388 & 5.93 \\
\hline "NS = not significant; & RR = relative risk. & & \\
\hline
\end{tabular}

\section{DISCUSSION}

\section{Prognosis and Surgical Resection}

There is broad agreement in the literature that radical surgery has a positive influence on the prognosis of benign and malignant meningiomas.[1,5,14,16,25,27,30,31,33,39] However, in most papers dealing with atypical and malignant meningiomas, radical surgery is largely understood to include both Simpson Grades I and II excision. The recurrence rate is reported to double for Simpson Grade II as opposed to Grade I excision in benign meningiomas,[14,33] evidently due to regrowth of neoplastic cells escaping dural coagulation. Regrowth of this kind in nonbenign meningioma after nonradical excision (Simpson Grade II) can reasonably be expected to be more frequent and faster. Controversial data on the prognostic differentiation between atypical and malignant meningiomas might in part be the result of the different distribution of Simpson Grades I and II excisions in the two groups. This is why we included only patients undergoing Simpson Grade I excision in the radical extirpation group. The clinical course of Simpson Grade II cases was similar to that of Grade III cases in our series, whereas Simpson Grade I patients fared significantly better (Fig. 5).

The favorable prognostic influence of radical surgery in our series is further emphasized by the zero recurrence rate observed in convexity anaplastic meningiomas treated in this way. The recurrences in parasagittal and basal tumors even after Simpson Grade I resection seem contradictory but can be explained by the fact that true Simpson Grade I resection can only be accomplished for tumors located in the cerebral convexity. In other locations, some neoplastic cells promoting tumor regrowth are always left behind because of the unfavorable surgical conditions intrinsic to the site. In a recent paper, Kinjo, et al.,[20] stressed the fact that only convexity meningiomas can be completely extirpated in all cases by enlarging the Simpson Grade I resection with the removal of an additional dural margin of approximately $2 \mathrm{~cm}$ around the tumor (so-called "grade zero removal") as suggested by Borovich, et al.,[3] to overcome the possible regional multicentricity of meningioma cells. In our experience, however, the traditional Simpson Grade I resection was enough to eradicate anaplastic meningiomas located in the cerebral convexity and procure a favorable long-term prognosis.

\section{Prognosis and Histology: the Problem of Atypical Meningiomas}

Atypical and malignant meningiomas remain a controversial topic because of the lack of universally accepted criteria for grading histological malignancy and because few large series have been conducted to evaluate the problem of malignancy.[2,4,6,9,12,13,15-17,23,24,26,34,39,41] Particular problems in this context are the meningiomas with limited signs of histological anaplasia, termed "atypical" and recently introduced into the $\mathrm{WHO}$ classification. This category is intended to constitute an intermediate 
group between benign and malignant meningiomas, with a supposedly intermediate prognosis.[21,40]

The results of our study showed that after a long-term follow-up period ranging from 8 to 34 years our series of 42 patients with atypical meningioma showed significantly better outcomes in terms of cumulative survival, recurrence-free survival, and median time to recurrence than 29 patients with malignant meningioma (Fig. 2-4). The late mortality rate due to recurrence in our series was also lower and the mean time to death longer for patients with atypical rather than malignant meningioma ( $31 \%$ and 10.5 years vs. $74 \%$ and 5.5 years, respectively). It should be noted, however, that when we consider only cases with incomplete resection (Simpson Grades II and III), the survival curve of atypical meningioma was not significantly better than that of malignant meningioma (Fig. 5). We also want to stress the great variability in the clinical courses of our patients with atypical meningioma; approximately $50 \%$ of them fared just as well as the benign group, whereas another $25 \%$ took the steep downhill course typical of the malignant group. The last $25 \%$ had a relatively long clinical history characterized by slow evolution toward frank malignancy and a fatal outcome after many recurrences over a period as long as 15 years, like the patients described by Cushing and Eisenhardt.[8] In our view, this last subgroup of cases are examples of "true" atypical meningiomas; unfortunately, we were unable to identify them in advance by their histopathological features. A review of the literature indicates that the atypical meningioma group is still poorly defined and that we are still far from being able to assess a priori the real prognostic influence of limited histological atypia in individual cases.[2,4,6,9,12,13,16,17,23,24,26,34,39,41] In considering the problem of the influence of malignant histological features on meningioma recurrences, many authors have failed to differentiate atypical from anaplastic (malignant) forms or have grouped them together to analyze histological elements common to both.[4,9,11,13,17,25,26,38] Zülch and Mennel[41] formulated the four-step grading scheme for meningiomas later adopted as the WHO classification, but grouped Grade II and III tumors together, stating that the prognostic significance of differentiating the two subgroups did not seem to be sufficiently established. More recently, Thomas, et al.,[34] reviewed 15 cases of malignant meningioma and concluded that there was no convincing difference in survival between cases of obvious and borderline malignancy (the latter probably including atypical meningiomas). Surprisingly, they found mitosis to be more indicative of malignancy than necrosis and brain invasion. They concluded that factors other than histological appearance determined outcome in cases of anaplastic meningeal tumors, but did not discuss the role of surgical excision. Jääskeläinen, et al.,[16] who created the scoring system we used to separate atypical from malignant meningiomas, observed a significantly higher recurrence rate for malignant than for atypical forms (78\% vs. 38\%) 5 years after "complete" resection, but failed to note differences between the two groups in terms of growth rate and median time to recurrence. They regarded Simpson Grades I and II as "complete resection," but in a later study Jääskeläinen[14] found that simple coagulation of the dura mater (Simpson Grade II) versus extirpation (Simpson Grade I) doubled the recurrence rate in that series of benign meningiomas.

Alvarez, et al.,[2] studied 21 cases of malignant and atypical meningiomas. Simpson Grade I resection was performed in 15 of 21 cases but the distribution of Grades II to IV resection between atypical and anaplastic (malignant) meningiomas was not specified. The criteria they used to separate the 15 cases regarded as atypical from the other six classified as malignant meningioma were based on the incidences in each case of the different histological features denoting anaplasia. A meningioma was labeled malignant if it had all the histological signs of malignancy (loss of architecture, high typical or atypical mitotic rate, necrosis, and brain invasion). Meningiomas with a high mitotic rate associated with at least one of the other histological hallmarks of anaplasia were diagnosed as atypical. As a result, these authors found a recurrence rate of $83.3 \%$ for malignant as compared to $25 \%$ for atypical meningioma after a 
mean follow-up period of 6.9 and 3.7 years, respectively. The late mortality rate was also apparently higher in the malignant meningioma (66.6\%) than in the atypical meningioma (33.3\%) group, in which, however, three intraoperative deaths were included. No conclusion can be drawn about a better prognosis for atypical meningioma with respect to malignant meningioma from the preceding study.

Mahmood, et al.,[23] reported their experience with 20 atypical and five malignant meningiomas in a consecutive series of 319 primary intracranial meningeal tumors. The scoring system used to classify the cases as atypical or malignant meningioma was similar to that of Jääskeläinen, et al.,[16] used in the present study, but even more accurate because the score for each parameter was based on objectively assigned values rather than subjective evaluation. In the few cases studied, these authors found no statistically significant differences between recurrence and survival rates for atypical and malignant meningiomas. They also noted that tumor recurrence was accompanied by dedifferentiation from a more benign histological finding in five cases.

In a recent paper by Maier, et al.,[24] 1799 meningiomas were studied retrospectively for correlations between histopathological subtypes and the recurrence rate. They classified surgical excision subjectively as total, subtotal, and partial: in $63 \%$ of their 35 cases of atypical meningioma and in $60 \%$ of the 40 malignant meningiomas total resection was reported. They stressed that a clinically relevant separation can be made between atypical and malignant meningeal tumors. In their study, atypical meningioma was simply defined on the basis of increased cellularity and at least five mitotic figures in $10 \mathrm{hpf}$, whereas malignant tumors contained all six of the features of anaplasia described in the WHO classification.[21] The recurrence rate for atypical and malignant meningiomas was found to be $34.6 \%$ and $72.6 \%$, respectively, and the authors concluded that atypical meningiomas, as defined in their study, did in fact have an intermediate prognosis with respect to benign and malignant tumors.

McLean, et al.,[26] analyzed the survival of 28 patients, 15 with atypical and 13 with malignant meningiomas as classified by the present WHO criteria. Their aim, however, was not to differentiate atypical from malignant meningiomas but rather to determine the prognostic importance of the individual histopathological features. They found that micronecrosis was the only prognostic indicator in the group of atypical/malignant meningiomas.

Younis, et al.,[39] recently reviewed a series of 25 aggressive meningeal tumors including 12 malignant and six atypical meningiomas. In their view the main features differentiating malignant from atypical meningiomas were brain invasion, sarcomatous areas, and "extreme degrees of the histological features associated with aggressive tumor behavior" according to WHO guidelines. However, they pointed out that histopathological differentiation in such terms is both subjective and difficult: surprisingly, they found that patients with atypical meningioma had shorter survival times than those with malignant meningioma. On the other hand, the survival time was longer after total than after partial resection. They concluded that because histological features alone can be unreliable in evaluating the aggressiveness of meningioma, it was advisable to incorporate proliferation indices into the diagnostic procedure. This conclusion is endorsed by us with the reservation that WHO histological criteria failed to differentiate prognostically a minority of atypical from malignant meningiomas in our series. We are therefore planning a retrospective study of proliferative indices in our atypical meningioma cases in search of a better correlation with the variable clinical course we observed. Biological indices of proliferative potential for the prognostic assessment of meningiomas have been the subject of many papers in recent years.[6,12,22,29,30,32,37] Parameters such as the cytogenetic pattern[22,37] and flow-cytometric analysis of DNA content,[30] and proliferation indices such as in situ bromodeoxyuridine 
incorporation,[32] Ki-67, and the proliferating cell nuclear antigen index[6,12,22,29,30] have been considered. The data acquired so far show a good but incomplete correlation between high proliferative index at initial presentation and malignant clinical behavior.[6,12,22,29,30,32] The group of atypical meningiomas was found to be heterogeneous even with respect to the biological markers of proliferative potential and showed a considerable overlap of values with benign and malignant meningiomas.[12,22,37] The systematic study of proliferation indices will presumably help to reveal potentially malignant tumors hitherto misclassified as atypical on the basis of traditional histology, thus improving their prognostic assessment.

\section{CONCLUSIONS}

The following conclusions emerge from the present study.

1) Atypical meningioma as defined by WHO criteria is a prognostically heterogeneous group with most patients living a long time (some of whom eventually fall victim to malignant transformation) and others surviving only a few years. Reports in the literature indicate that routine evaluation of proliferative potential and the cytogenetic pattern of resected tumors will eventually help to determine with more accuracy the prognosis for atypical meningiomas.

2) Simpson Grade I extirpation can be performed reliably in convexity meningiomas and may be successful despite malignant histological findings.

\section{References}

1. Adegbite AB, Khan MI, Paine KWE, et al: The recurrence of intracranial meningiomas after surgical treatment. J Neurosurg 58:51-56, 1983

2. Alvarez F, Roda JM, Romero MP, et al: Malignant and atypical meningiomas: a reappraisal of clinical, histological, and computed tomographic features. Neurosurgery 20:688-694, 1987

3. Borovich B, Doron Y, Braun Y, et al: Recurrence of intracranial meningiomas: the role played by regional multicentricity. Part 2: Clinical and radiological aspects. J Neurosurg 65:168-171, 1986

4. Chen WY, Liu HC: Atypical (anaplastic) meningioma: relationship between histologic features and recurrence--a clinicopathological study. Clin Neuropathol 9:74-81, 1990

5. Christensen D, Laursen H, Klinken L: Prediction of recurrence in meningiomas after surgical treatment. A quantitative approach. Acta Neuropathol 61:130-134, 1983

6. Cobb MA, Husain M, Andersen BJ, et al: Significance of proliferating cell nuclear antigen in predicting recurrence of intracranial meningioma. J Neurosurg 84:85-90, 1996

7. Cox DR: Regression models and life tables. J R Stat Soc 34:187-220, 1972

8. Cushing HW, Eisenhardt L: Meningiomas: Their Classification, Regional Behavior, Life History, and Surgical End Results. Springfield, Ill: Charles C. Thomas, 1938, pp 47-50, 669-719

9. DelaMonte SM, Flickinger J, Linggood RM: Histopathological features predicting recurrence of meningiomas following subtotal resection. Am J Surg Pathol 10:836-843, 1986

10. Dixon WJ (ed): BMDP Statistical Software. Berkley, CA: University of California Press, 1993 
11. Fabiani A, Trebini F, Favero M: The significance of atypical mitosis in malignant meningiomas. Acta Neuropathol 38:229-231, 1977

12. Hsu DW, Pardo FS, Efird JT, et al: Prognostic significance of proliferative indices in meningiomas. J Neuropathol Exp Neurol 53:247-255, 1994

13. Inoue H, Tamura H, Koizumi H, et al: Clinical pathology of malignant meningiomas. Acta Neurochir 73:179-191, 1984

14. Jääskeläinen J: Seemingly complete removal of histologically benign intracranial meningioma: late recurrence rate and factors predicting recurrence in 657 patients. A multivariate analysis. Surg Neurol 26:461-469, 1986

15. Jääskeläinen J, Haltia M, Laasonen E, et al: The growth rate of intracranial meningiomas and its relation to histology. An analysis of 43 patients. Surg Neurol 24:165-172, 1985

16. Jääskeläinen J, Haltia M, Servo A: Atypical and anaplastic meningiomas: radiology, surgery, radiotherapy, and outcome. Surg Neurol 25:233-242, 1986

17. Jellinger K, Slowik F: Histological subtypes and prognostic problems in meningiomas. J Neurol 208:279-298, 1975

18. Kaplan EL, Meier P: Nonparametric estimation from incomplete observations. J Am Stat Assoc 53:457-481, 1958

19. Kepes JJ: Comment on Mahmood A, Caccamo DV, Tomecek FJ, et al: Atypical and malignant meningiomas: a clinicopathological review. Neurosurgery 33:963, 1993

20. Kinjo J, Al-Mefty O, Kanaan I: Grade zero removal of supratentorial convexity meningiomas. Neurosurgery 33:394-399, 1993

21. Kleihues P, Burger PC, Scheithauer BW: Histological Typing of Tumours of the Central Nervous System, ed 2. Berlin: Springer-Verlag, 1993, pp 36-37

22. Kolles H, Niedermayer I, Schmitt C, et al: Triple approach for diagnosis and grading of meningiomas: histology, morphometry of Ki-67/Feulgen stainings, and cytogenetics. Acta Neurochir 137:174-181, 1995

23. Mahmood A, Caccamo DV, Tomecek FJ, et al: Atypical and malignant meningiomas: a clinicopathological review. Neurosurgery 33:955-963, 1993

24. Maier H, Öfner D, Hittmair A, et al: Classic, atypical, and anaplastic meningioma: three histopathological subtypes of clinical relevance. J Neurosurg 77:616-623, 1992

25. Marks SM, Whitwell HL, Lye RH: Recurrence of meningiomas after operation. Surg Neurol 25:436-440, 1986

26. McLean CA, Jolley D, Cukier E, et al: Atypical and malignant meningiomas: importance of micronecrosis as a prognostic indicator. Histopathology 23:349-353, 1993

27. Mirimanoff RO, Dosoretz DE, Linggood RM, et al: Meningioma: analysis of recurrence and progression following neurosurgical resection. J Neurosurg 62:18-24, 1985 
28. Palma L, Celli P, Cervoni N, et al: Atypical and malignant meningiomas: clinico-pathological study of 111 cases operated on. Clin Neuropathol 11:213, 1992 (Abstract)

29. Roggendorf W, Schuster T, Pfeiffer J: Proliferative potential of meningiomas determined with the monoclonal antibody Ki-67. Acta Neuropathol 73:361-364, 1987

30. Salmon I, Kiss R, Levivier M, et al: Characterization of nuclear DNA content, proliferation index, and nuclear size in a series of 181 meningiomas, including benign primary, recurrent, and malignant tumors. Am J Surg Pathol 17:239-247, 1993

31. Scheithauer BW: Tumors of the meninges: proposed modifications of the World Health Organization classification. Acta Neuropathl 80:343-354, 1990

32. Shibuya M, Hoshino T, Ito S, et al: Meningiomas: clinical implications of a high proliferative potential determined by bromodeoxyuridine labeling. Neurosurgery 30:494-498, 1992

33. Simpson D: The recurrence of intracranial meningiomas after surgical treatment. J Neurol Neurosurg Psychiatry 20:22-39, 1957

34. Thomas HG, Dolman CL, Berry K: Malignant meningioma: clinical and pathological features. J Neurosurg 55:929-934, 1981

35. Turner OA, Craig WM, Kernohan JW: Malignant meningiomas. A clinical and pathologic study. Surgery 11:81-100, 1942

36. Tytus JS, Lasersohn JT, Reifel E: The problem of malignancy in meningiomas. J Neurosurg 27:551-557, 1967

37. Vagner-Capodano AM, Grisoli F, Gambarelli D, et al: Correlation between cytogenetic and histopathological findings in 75 human meningiomas. Neurosurgery 32:892-900, 1993

38. Wilson CB: Meningiomas: genetics, malignancy and the role of radiation in induction and treatment. The Richard C. Schneider Lecture. J Neurosurg 81:666-675, 1994

39. Younis GA, Sawaya R, De Monte F, et al: Aggressive meningeal tumors: review of a series. J Neurosurg 82:17-27, 1995

40. Zülch KJ (ed): Histological Typing of Tumours of the Central Nervous System. International Classification of Tumours, No. 21. Geneva: World Health Organization, 1979

41. Zülch KJ, Mennel HD: Malignant meningiomas. Adv Neurosurg 2:3-11, 1975

Manuscript received January 28, 1997.

Accepted in final form March 17, 1997.

Address reprint requests to: Lucio Palma, M.D., Department of Ophthalmological and Neurosurgical Sciences, Siena University Medical School, Le Scotte Hospital, Viale Bracci, 1-53100 Siena, Italy. 